\title{
Demagnetization of Current Transformers Using PWM Burden
}

\author{
Jan Bauer ${ }^{1}$, Pavel Ripka ${ }^{1}$, Karel Draxler ${ }^{1}$, and Renata Styblikova ${ }^{2}$ \\ ${ }^{1}$ Faculty of Electrical Engineering, Czech Technical University, Prague 166 36, Czech Republic \\ ${ }^{2}$ Czech Metrology Institute, Prague 638 00, Czech Republic
}

Current transformers (CTs) show large errors when they are magnetized by dc current. This error can be reduced after proper demagnetization. One of the methods to demagnetize the CT is to increase the core flux by increasing its burden. The burden should be adjusted according to the measured ac current. In this paper, we show that pulsewidth modulation switchable resistor can be used as variable burden for this application. This method enables to restore the nominal precision of the heavily magnetized CT from $2.5 \%$ back to $0.2 \%$ without interruption of the CT operation.

Index Terms-Current transformer (CT), lock-in amplifier, pulsewidth modulation (PWM).

\section{INTRODUCTION}

$\mathbf{C}$ URRENT transformers (CTs) are susceptible to remanence caused by dc magnetization. In such case, the precision of the CT can be seriously degraded especially at low measured currents [1]-[4]. Magnetization can be caused by temporary unipolar transients from lightning or switching of power devices to the supply grid. When the CT operates at $>80 \%$ of its nominal current $I_{N}$, the CT is spontaneously demagnetized. For lower measured ac current the CT remains permanently magnetized. This effect can cause large errors especially when measuring energy transferred in unloaded supply network. The origin of this error is that magnetized core has lower apparent permeability [2].

Method for measuring the dc component of the current using fuxgate effect in the $\mathrm{CT}$ was described in [5]-[7]. The dc current can be compensated to restore the CT precision [8], [9]. These methods can also be used to demagnetize the $\mathrm{CT}$ and keep it without remanence. However, the instrumentation is complicated and requires an additional source of power [10]. The aim of this paper is to develop a simple device for demagnetizing the CTs in the feld.

The magnetized CT can be demagnetized by increasing the measured current, e.g., to $120 \%$ of the nominal value $I_{N}$ and slowly decreasing it to zero. This is easy in the lab, but not very practical when implemented in a network. Another technique consists in increasing the burden so as to increase the voltage at the ends of the secondary winding and therefore the magnetic fux in the core. For a measured current of $0.1 I_{N}$ the required increase or the burden is theoretically 12 times $R_{N}$. The transient from switching the burden back
to $R_{N}$ should be controlled to prevent another magnetization. In some cases this is not trivial.

This paper is organized as follows. In Section II, we fir $t$ show that by increased burden we are able to effectively demagnetize $\mathrm{CT}$ so that resulting error is very similar to CT demagnetized by conventional method.

Then, we describe and discuss two methods how to adjust $R_{2}$. Section III describes in detail the principle of cvut.cz).

I]

Color versions of one or more of the f gures in this paper are available online at http://ieeexplore.ieee.org.

Digital Object Identifie 10.1109/TMAG.2014.2356574

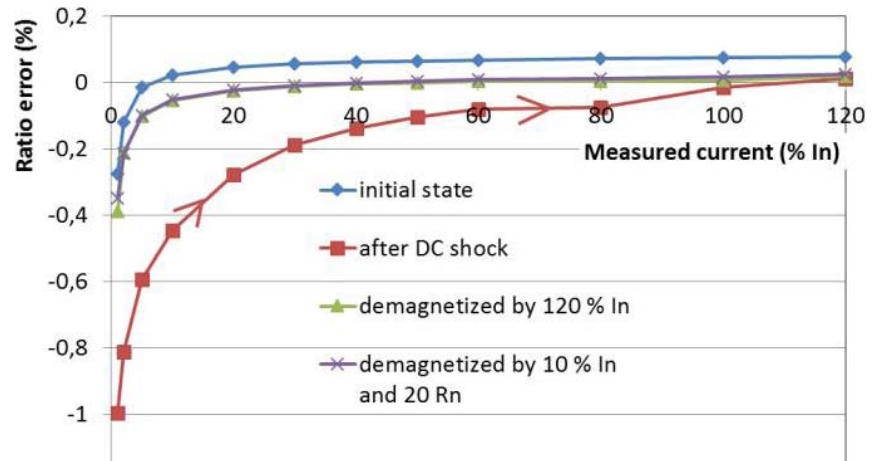

Fig. 1-1, Ratio error of $500 \mathrm{~A} / 5 \mathrm{~A} \mathrm{CT}$ in the virgin state and after magnetization to remanence and two methods of demagnetization.

Fig. 2. Resistor network for increas $\mathrm{R}_{1}$ f burden. $\mathrm{R}_{2}$

the pulsewidth modulation (PWM) switched resistor method, which was selected for our device. In Section IV, we experimentally verify the proposed method.

\section{Demagnetization Methods}

Fig. 1 shows the ratio (amplitude) error of the class $0.2 \mathrm{CT}$ with $500 \mathrm{~A} / 5 \mathrm{~A}$ ratio. The core of this transformer is made of high-permeability oriented Si-Fe. The perfectly demagnetized CT has ratio error $<0.2 \%$ in the wide range from $2 \%$ to $120 \% I_{N}$. After magnetization by large dc current to the remanence this transformer is out of its accuracy class for $I<30 \% I_{N}$. Only after $I>80 \% I_{N}$, the core slowly starts to demagnetize. We magnetized the transformer again and demagnetized it by manually increasing the burden to $20 R_{N}$ at $10 \% I_{N}$. The quality of demagnetization is very similar as before; although it is not ideal, the CT specs are again met.

For real application $R_{2}$ should be adjusted according to the actual value of $I_{1}$. We can either use conventional switched network of weighed resistors (Fig. 2), or PWM of switched resistor (Fig. 3).

Resistor network is a simple solution, but it has a substantial problem for fi e resistance adjustment it needs six to eight switches, which brings large uncertainty caused by 


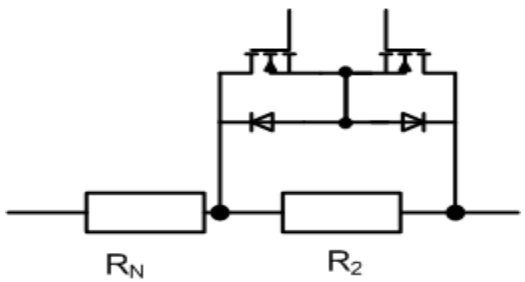

Fig. 3. PWM switched resistor.

switch ON-resistances. The second method requires more complicated control logic, but allows f ne adjustment with single switch. In this paper, we therefore verify the demagnetization by PWM switched resistor.

\section{PWM SWITCHED RESISTOR}

The development of the semiconductor devices and improvement of their parameters have already influence area of electric drives and energy conversion. However, a limiting parameter of these devices is the amount of heat that needs to be dissipated because the heat is proportional to the power losses caused mainly during the switching transition and the inner resistance of the semiconductor, switching of these devices was limited. Recent development in the area of MOS transistors has led to devices with very low resistance $r_{\text {DSon }}$ in the range of $10 \mathrm{~m} \Omega$. Such devices can be used in circuits, where power electronics is needed and its infl ence should be negligible.

As stated previously, the burden value can be increased either by a resistor network or by PWM switched resistor. First, solution is simpler but requires more space and continuous change of the resistance is not possible. Therefore, we have focused on the PWM switched resistor solution. The PWM controlled variable resistor is already used in [11] as a damping resistor for protecting the power capacitor/passive power flter.

Because the secondary winding of the CT cannot be unloaded the solution shown in Fig. 2 was selected for PWM controlled resistor realization. The scheme is the same as in [12], but the switching scheme is different, whereas in [12] the switching time is the multiple of power line frequency, we used much higher frequency. The solution in [12] was developed for protective CTs, where accuracy is not critical parameter. Our solution was developed for instrument CTs.

In normal operation mode, both transistors are switched ON and the secondary current is according to its polarity fowing through one transistor and one antiparallel diode. When demagnetization of the CT is needed, PWM is enabled and transistors begin to be switched. Current then fows through the transistor when it is switched ON or through increased burden when the transistor is switched OFF.

The principle of PWM is well known from the semiconductor power converters, where PWM is employed for the generation of voltages with variable frequency and mean value. The mean value of the output voltage across one period can be calculated as

$$
\bar{v}=\frac{1}{T_{S}} \int_{0}^{T_{s}} v(t) d t
$$

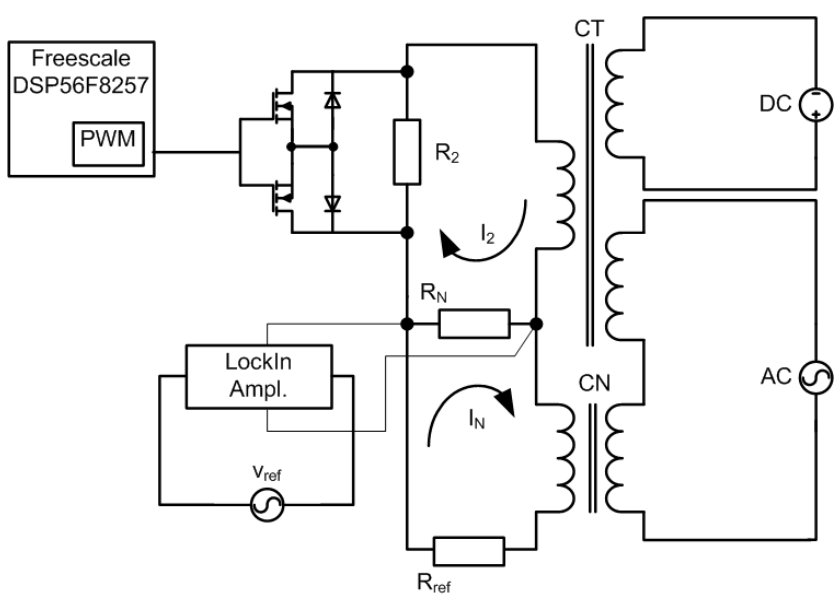

Fig. 4. Layout of the demagnetization device. CT converts the ac primary current $I_{1}$ into the secondary current $I_{2}$. CT is magnetized by momentary dc current $I_{\mathrm{dc}}$ injected into the auxiliary winding.

When we consider PWM principle and replace voltage by $R(t)$ that has value of $r_{\mathrm{DSon}}$ for $t \in\left[0, t_{\mathrm{ON}}\right]$ and $R_{2}$ for $t \in\left[t_{\mathrm{ON}}, T_{S}\right]$, we can rewrite (1) into

$$
\begin{aligned}
\bar{R} & =\frac{1}{T_{S}} \int_{0}^{T_{S}} R(t) d t=\frac{1}{T_{s}}\left(\int_{0}^{t_{\mathrm{ON}}} r_{\mathrm{DS}(\mathrm{ON})} d t+\int_{t_{\mathrm{ON}}}^{T_{S}} R_{2} d t\right) \\
& =\frac{t_{\mathrm{On}}}{T_{S}} r_{\mathrm{DS}(\mathrm{ON})}+\frac{t_{\mathrm{OFF}}}{T_{S}} R_{2}
\end{aligned}
$$

where $t_{\mathrm{OFF}}=T_{S}-t_{\mathrm{ON}}$.

Because the $r_{\text {DSon }}$ can be connected to the circuit during the normal operation of the CT, it can be included in the value of the burden. Equation (2) will be therefore simplif ed as

$$
\bar{R}=\frac{t_{\mathrm{OFF}}}{T_{S}} R_{2} \text {. }
$$

If the switching period $T_{S}$ is several times shorter than the period of the measured current signal, the switching transition of the transistors can be neglected and the value of $R_{2}$ can be calculated according to (3). Equation (3) then define the value of equivalent resistance connected in series with the burden to demagnetize the transformer.

In analog technique, the PWM modulator can be realized as an operational amplif er that is comparing triangular carrier with variable threshold value. However, analog devices suffer from ageing, this is why analog solutions are replaced by digital solutions in a microprocessor.

\section{EXPERIMENTAL VALIDATION OF THE}

\section{DEMAGNETIZATION OF A CT USING A PWM BURDEN}

For our experiment, we used a $\mathrm{CT}$ with transformation ratio of $500 \mathrm{~A} / 5 \mathrm{~A}$ and real $5 \mathrm{VA}$ nominal secondary burden, corresponding to a resistor of $0.2 \Omega$. The output of the transformer was loaded by sensing resistor $R_{N}=0.1 \Omega$. A VISHAY MPR resistor with value of $2 \Omega$ was used as PWM switched resistor $R_{2}$. The CT errors were measured using a lock-in amplifie. Switching pulses for the transistors were generated by Freescale DSP56F8257 microcontroller. The period of PWM $T_{\mathrm{CS}}$ was $5 \mathrm{~ms}$.

The experimental setup shown in Fig. 4 is used to implement the proposed CT demagnetization method and to determine the 
TABLE I

MEASUREd CT ERRORS

\begin{tabular}{lcc}
\hline \hline \multicolumn{1}{c}{ State of CT } & Ratio error (\%) & Phase displacement (“) \\
\hline Initial error & -0.24 & -44 \\
for $\mathrm{I}_{2}=1 \mathrm{~A}$ & & \\
Magnetized CT & 2.50 & -172 \\
Demagnetization for & -0.15 & -47 \\
$\mathrm{R}_{2}=2 \Omega$ & & \\
Magnetized CT & 2.28 & -190 \\
Demagnetization & -0.12 & -47 \\
by increased current & & \\
$\mathrm{I}_{2}=6 \mathrm{~A}$ & & \\
Initial error & -0.29 & -34 \\
\hline for $\mathrm{I}_{2}=0.5$ A & & \\
Magnetized CT & 4.66 & -273 \\
Demagnetization by & 1.18 & -82 \\
$\mathrm{R}_{2}=2 \Omega$ & & -47 \\
Demagnetization by & -0.3 & \\
$\mathrm{R}_{2}=12 \Omega$ & & \\
\hline
\end{tabular}

errors on the measured currents. The principle of the experiments basically relies on the use of a differential structure implementing a CT under test (denoted CT) as well as a reference $\mathrm{CT}$ (denoted $\mathrm{CN}$ ) with very high accuracy. In the experimental setup only CT may be submitted to a dc magnetization of its core. Therefore, an auxiliary winding featuring 50 turns and fed by a dc current is used. To demagnetize CT, its secondary winding features a PWM switched resistor of equivalent value in series with a resistance $R_{N}$. The ac current to measure feeds both the primary windings of $\mathrm{CT}$ and $\mathrm{CN}$. The secondary windings of CT and $\mathrm{CN}$ (which are wound in opposite directions) feed $R_{N}$ with opposite currents of similar intensity and the voltage $V_{N}$ at the ends of $R_{N}$ is proportional to the difference between these two currents. The CV amplitude and phase error is therefore determined from the measurement (performed by lock-in amplifier of the voltage drop of $V_{N}$. More detailed description of the method used for CT error measurement and test bed can be found in [13]. The layout of the demagnetization device is shown in Fig. 3.

According to [13], the ratio error of measured current amplitude can be calculated as real part of the voltage $\Delta V$ measured by a lock-in amplifie as

$$
\varepsilon_{I}=\frac{\operatorname{Re}(\Delta I)}{I_{N}}=\frac{\operatorname{Re}(\Delta V)}{R_{N}} \cdot \frac{R_{\mathrm{ref}}}{V_{\text {ref }}} \cdot 100(\%) .
$$

The error of measured current phase can be obtained from imaginary part of voltage as

$$
\delta_{I}=\frac{\operatorname{Im}(\Delta I)}{I_{N}}=\frac{\operatorname{Im}(\Delta V)}{R_{N}} \cdot \frac{R_{\mathrm{ref}}}{V_{\mathrm{ref}}} \cdot(\mathrm{rad})
$$

where $V_{\text {ref }}$ is a voltage measured across the $R_{\text {ref }}$.

The measurement results are summarized in Table I.

The measurements were performed at two CT operation points: 1) the CT was measuring at $20 \%$ of its nominal current $I_{N}$ and 2) at $10 \%$ of $I_{N}$. This corresponds to state of non-loaded network. First, the ratio error and phase displacement in initial state were measured. Then, the CT was

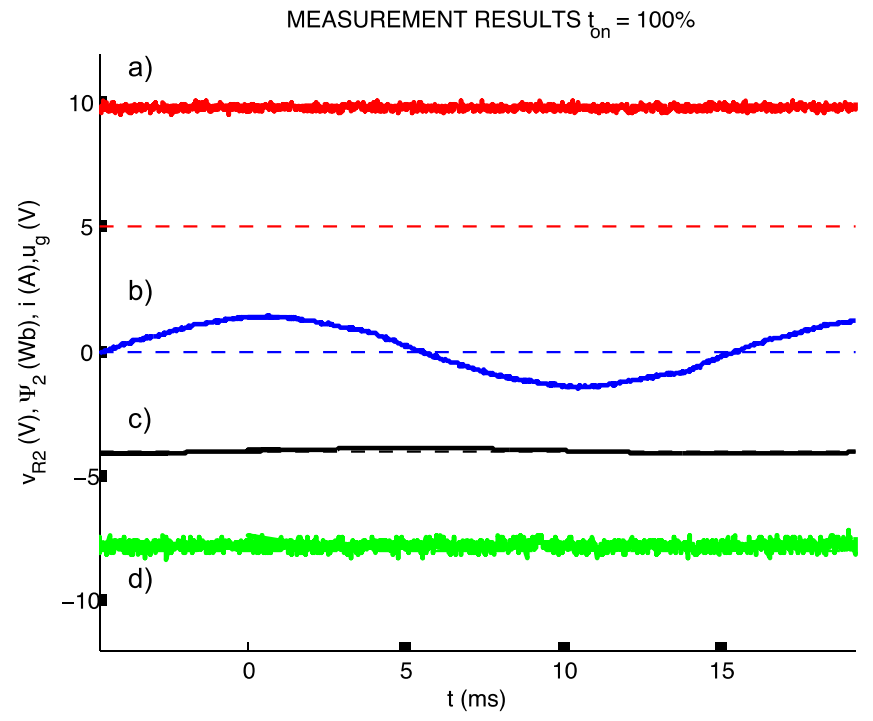

Fig. 5. Waveforms for $t_{\mathrm{ON}}=100 \%$. (a) Control signal for the transistor. (b) Secondary current of CT. (c) CT flux (d) Secondary voltage of CT.

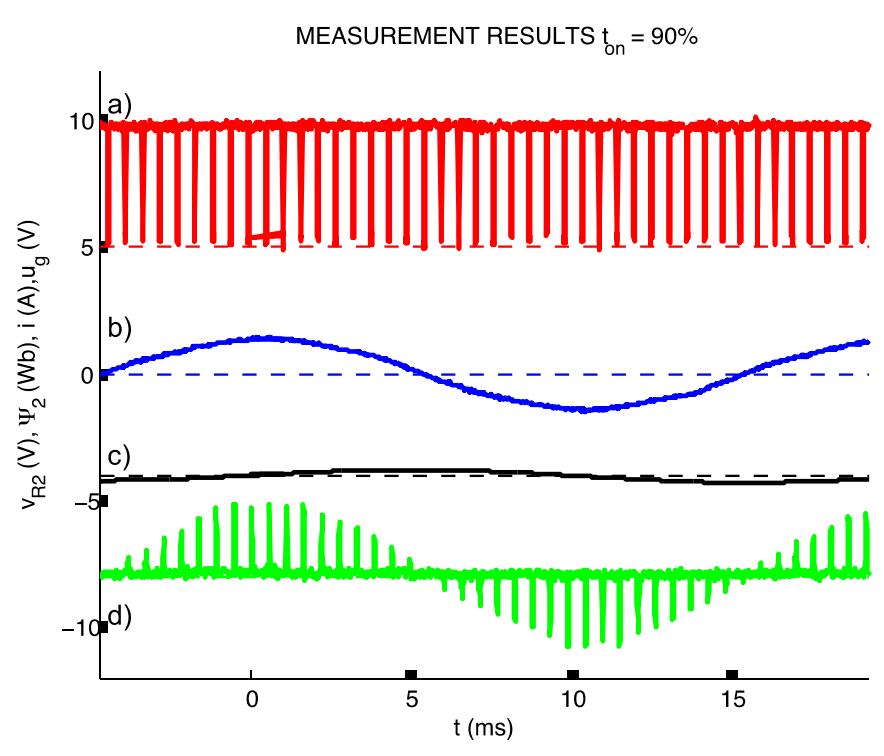

Fig. 6. Waveforms for $t_{\mathrm{ON}}=90 \%$. (a) Control signal for the transistor. (b) Secondary current of CT. (c) CT flux (d) Secondary voltage of CT.

magnetized by de current pulse of $10 \mathrm{~A}$ into the auxiliary winding of 50 turns and the errors were measured again. After that the demagnetization by PWM switched resistor was performed and the CT errors were measured once more. Both methods of demagnetization by increased input current and by PWM switched resistor were used. The results in Table I show that for $10 \%$ of the nominal current $I_{N}$ the value of $R_{2}=2 \Omega$ was not enough to fully CT demagnetization. The $R_{2}$ have to be increased.

Waveforms taken during the measurement are shown in Figs. 5-8. Upper trace (a) represents control signal for the transistors $\left(u_{g}\right)$, second trace (b) is the CT secondary current $(i)$, third trace $(\mathrm{c})$ is the $\mathrm{CT}$ fl $\mathrm{x}\left(\Psi_{2}\right)$ calculated as an integral of the CT secondary voltage, and bottom trace (d) is the voltage across the resistor $R_{2}\left(u_{R 2}\right)$. The waveforms show that 


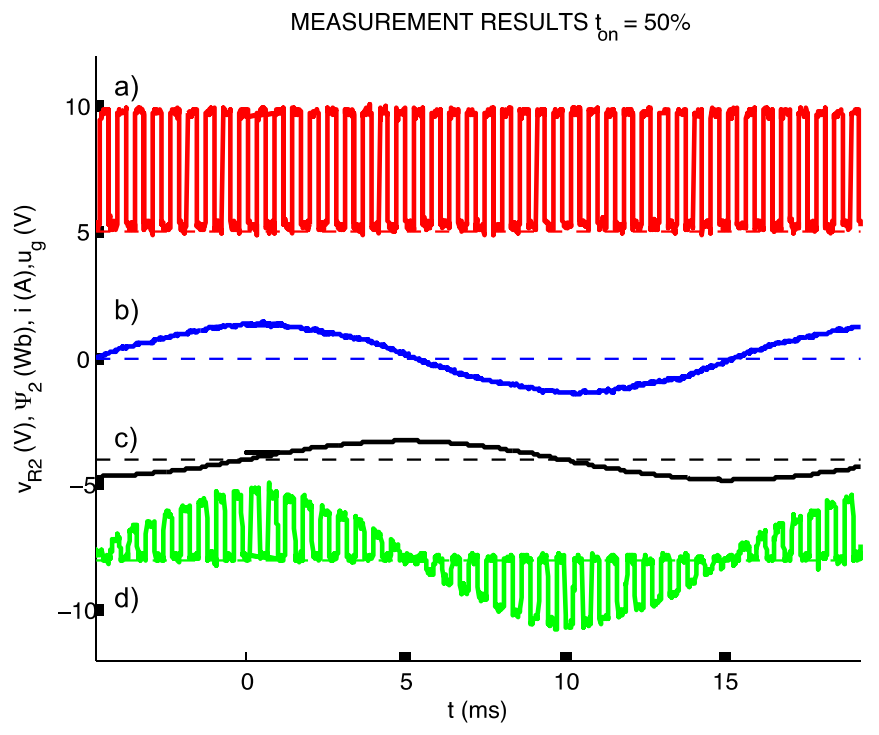

Fig. 7. Waveforms for $t_{\mathrm{ON}}=50 \%$. (a) Control signal for the transistor. (b) Secondary current of CT. (c) CT flux (d) Secondary voltage of CT.

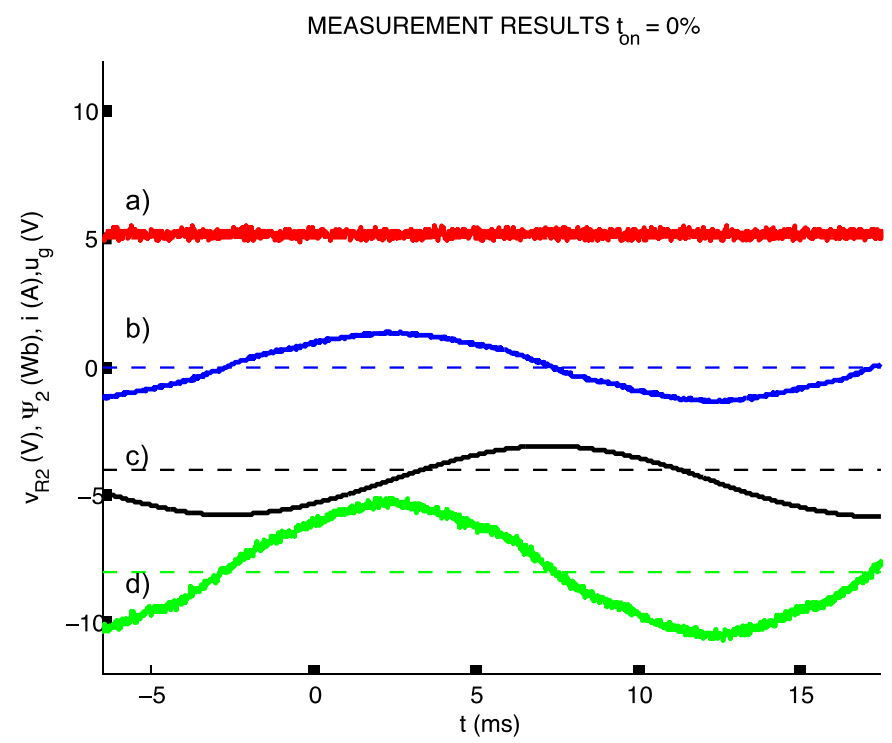

Fig. 8. Waveforms for $t_{\mathrm{ON}}=0 \%$. (a) Control signal for the transistor. (b) Secondary current of CT. (c) CT flux (d) Secondary voltage of CT.

switching frequency of the PWM was selected suff ciently high so that the CT fux is very close to sinewave, no distortion is visible.

\section{CONCLUSION}

The dc magnetization has great influenc on the CT accuracy, therefore demagnetization of $\mathrm{CT}$ is needed especially for high-accuracy CTs used for the energy meters. One way to demagnetize the $\mathrm{CT}$ is to increase the input current and thus saturate the core, however, this method is not possible in real application when CT is connected to the supply network. That is why the second method based on momentary increase of the load of the CT was tested. This can be done either by the resistor network or by PWM switching of a single resistor.

The main advantage of the PWM switched resistor is that compared with resistor network it enables possibility to use one resistor in the whole range of current and the value of increased load is controlled just by the means of PWM that allows continuous control of the load.

We have demonstrated that using this method it is possible to restore heavily magnetized $0.2 \%$ accuracy class CT back to its nominal error without removing it from the network.

During a practical use in an electrical network, the PWM switched resistor should be actuated to demagnetize the core typically in $15 \mathrm{~min}$ intervals and every time after the detected transient. The demagnetization phase should last about $10 \mathrm{~s}$, because of magnetic viscosity of the core material.

\section{REFERENCES}

[1] A. Braun, "Determination of current transformer errors at primary currents up to 100000 A," IEEE Trans. Instrum. Meas., vol. 26, no. 3, pp. 263-267, Sep. 1977.

[2] K. Draxler and R. Stybliková, "Effect of magnetization on instrument transformers errors," J. Elect. Eng., vol. 61, no. 7S, pp. 50-53, 2010.

[3] A. Rezaei-Zare, R. Iravani, M. Sanaye-Pasand, H. Mohseni, and S. Farhangi, "An accurate current transformer model based on Preisach theory for the analysis of electromagnetic transients," IEEE Trans. Power Del., vol. 23, no. 1, pp. 233-242, Jan. 2008.

[4] B. Wrzecionko, L. Steinmann, and J. W. Kolar, "High-bandwidth hightemperature $\left(250^{\circ} \mathrm{C} / 500^{\circ} \mathrm{F}\right)$ isolated $\mathrm{DC}$ and $\mathrm{AC}$ current measurement: Bidirectionally saturated current transformer," IEEE Trans. Power Electron., vol. 28, no. 11, pp. 5404-5413, Nov. 2013.

[5] G. Buticchi, E. Lorenzani, and G. Franceschini, "A DC offset current compensation strategy in transformerless grid-connected power converters," IEEE Trans. Power Del., vol. 26, no. 4, pp. 2743-2751, Oct. 2011

[6] C.-S. Yu, "Detection and correction of saturated current transformer measurements using decaying DC components," IEEE Trans. Power Del., vol. 25 , no. 3, pp. 1340-1347, Jul. 2010.

[7] P. Ripka, K. Draxler, and R. Styblikova, "Measurement of DC currents in the power grid by current transformer," IEEE Trans. Magn., vol. 49, no. 1, pp. 73-76, Jan. 2013.

[8] P. Stachel and P. Schegner, "Detection and correction of current transformer saturation effects in secondary current signals," in Proc. IEEE Power Energy Soc. General Meeting (PES), Jul. 2009, pp. 1-6.

[9] P. Ripka, K. Draxler, and R. Styblikova, "DC-compensated current transformer," in Proc. IEEE Int. Instrum. Meas. Technol. Conf., May 2014, pp. $212-215$.

[10] V. Molcrette, J.-L. Kotny, J.-P. Swan, and J.-F. Brudny, "Reduction of inrush current in single-phase transformer using virtual air gap technique," IEEE Trans. Magn., vol. 34, no. 4, pp. 1192-1194, Jul. 1998.

[11] J.-C. Wu, H.-L. Jou, and K.-D. Wu, "A PWM controlled variable damping resistor for protecting the power capacitor/passive power $\mathrm{flter}$," Electr. Power Syst. Res., vol. 73, no. 1, pp. 9-18, 2005.

[12] M. Davarpanah, M. Sanaye-Pasand, and R. Iravani, "A saturation suppression approach for the current transformer-Part I: Fundamental concepts and design," IEEE Trans. Power Del., vol. 28, no. 3, pp. 1928-1935, Jul. 2013.

[13] K. Draxler and R. Styblikova, "Use of a lock-in amplif er for calibrating an instrument current transformer," in Proc. IEEE Int. Instrum. Meas. Technol. Conf., May 2014, pp. 732-735. 